\title{
A Diagnostic Framework for Wind Turbine Gearboxes Using Machine Learning
}

\author{
Sofia Koukoura ${ }^{1}$, James Carroll ${ }^{2}$, and Alasdair McDonald ${ }^{3}$ \\ 1,2,3 University of Strathclyde, Glasgow, G1 1XQ,UK \\ sofia.koukoura@strath.ac.uk \\ j.carroll@strath.ac.uk
}

\begin{abstract}
Operation and maintenance costs of wind turbines are highly driven by gearbox failures, especially offshore were the logistics of replacements are more demanding. It is therefore very critical to foresee incipient gearbox faults before they become catastrophic failures. Wind turbine gearbox condition monitoring is usually performed using vibration signals coming from accelerometers installed on the gearbox surface. The current monitoring practice is a rule-based approach, where alarms are activated based on thresholds. However, too much manual analysis may be required for some failure modes and this can become quite challenging as the installed wind capacity grows. Also, since false alarms have to be avoided, these thresholds are set quite high, resulting in late stage diagnosis of components. Given the fact there is a large amount of historic operating data with confirmed gearbox failure incidents, this paper proposes a framework that uses a machine learning approach. Vibration signals are used from the gearbox sensors and processed in the frequency domain. Features are extracted from the processed signals based on the fault locations and failure modes, using domain knowledge. These features are used as inputs in a layer of pattern recognition models that can determine a potential component fault location and failure mode. The proposed framework is illustrated using failure examples from operating offshore wind turbines.
\end{abstract}

\section{INTRODUCTION}

The cost of energy of wind power generation needs to be driven down, and a large proportion of it consists of Operation and Maintenance (O\&M) costs. Wind turbines are designed to operate for around 25 years, however premature component failures occur, leading to increased downtime and loss of revenue. Conventional onshore O\&M activities consist of a combination of preventive and corrective maintenance operations, but this approach has room for im-

\footnotetext{
Sofia Koukoura et al. This is an open-access article distributed under the terms of the Creative Commons Attribution 3.0 United States License, which permits unrestricted use, distribution, and reproduction in any medium, provided the original author and source are credited.
}

provement (Artigao, Martín-Martínez, Honrubia-Escribano, \& Gómez-Lázaro, 2018).In offshore wind farms there is often lack of accessibility. Therefore, optimisation of maintenance actions need to be optimised and incipient component failures need to be foreseen in an efficient way. This can be achieved through predictive maintenance.

In recent years, Condition Monitoring Systems (CMS) systems have been incorporated in wind turbines and their efficiency has been improved. The main Condition Monitoring (CM) techniques along with the signal processing methods used for diagnosis and their applications in wind power are presented in (Hameed, Hong, Cho, Ahn, \& Song, 2009), (Amirat, Benbouzid, Al-Ahmar, Bensaker, \& Turri, 2009), (Márquez, Tobias, Pérez, \& Papaelias, 2012). The most popular technologies are vibration analysis and oil analysis.

A comprehensive reliability review was carried out in (Artigao et al., 2018), where all wind turbine failure statistics datasets are compared and analysed, to give meaningful conclusions for all types of wind turbines. According to the findings of this study, the gearbox shows the highest downtime. It is therefore imperative that condition monitoring strategies for wind turbine gearboxes are improved.

The current monitoring practice is a rule-based approach, where alarms are activated based on thresholds. However, too much manual analysis may be required for some failure modes and this can become quite challenging as the installed wind capacity grows. Also, since false alarms have to be avoided, these thresholds are set quite high, resulting in late stage diagnosis of components. As the installed wind capacity grows, so does the volume of condition monitoring data. In the era of Big Data and Internet of Things, there are plenty of Artificial Intelligence (AI) techniques that can be utilised by the wind industry to improve asset management. Given the fact there exists a large amount of historic operating data with confirmed gearbox failure incidents, this paper proposes a framework that uses a machine learning approach.

The rest of the paper is organised as follows: Section 2 


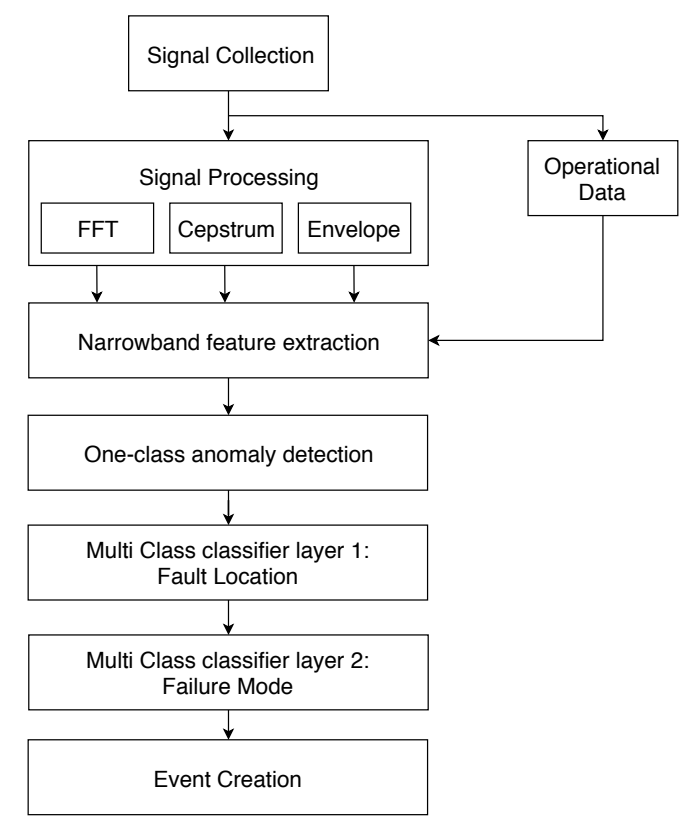

Figure 1. Wind Turbine Gearbox Diagnostic Framework

presents the proposed framework; the gearbox structure and kinematic data are given in 2 , the various signal processing methods for vibrations are presented in 2.2 , the features extracted from the signals are shown in 2.3 and these features are used as inputs in pattern recognition models as discussed in 2.4. A case study using operational data from wind turbines is given in 3 .

\section{FRAMEWORK}

The framework proposed in this paper is depicted in Figure 1. The steps include signal collection, signal processing, feature extraction and use of a serious of layers of pattern recognition models for anomaly detection, fault isolation and failure mode analysis.

\subsection{Gearbox Kinematic Data}

A gearbox is typically used in a wind turbine to increase rotational speed from a low-speed rotor to a higher speed electrical generator. Most existing operating wind turbines (up to $6 \mathrm{MW}$ ) have a conventional high speed geared drivetrain with an overall ratio of around 100:1. The gearboxes in these configurations have a one or two planetary stages and one or two parallel stages. A typical wind turbine gearbox configuration is shown in Figure 2. The framework presented will focus on this type of gearbox, however the proposed methodology can be applied to any other type.

Fault characteristic frequency is a widely useful fault characterizing feature in rotating machine fault diagnosis, because the fault induced features repeat themselves periodically with rotational frequency. As for the gearbox, the meshing fre-

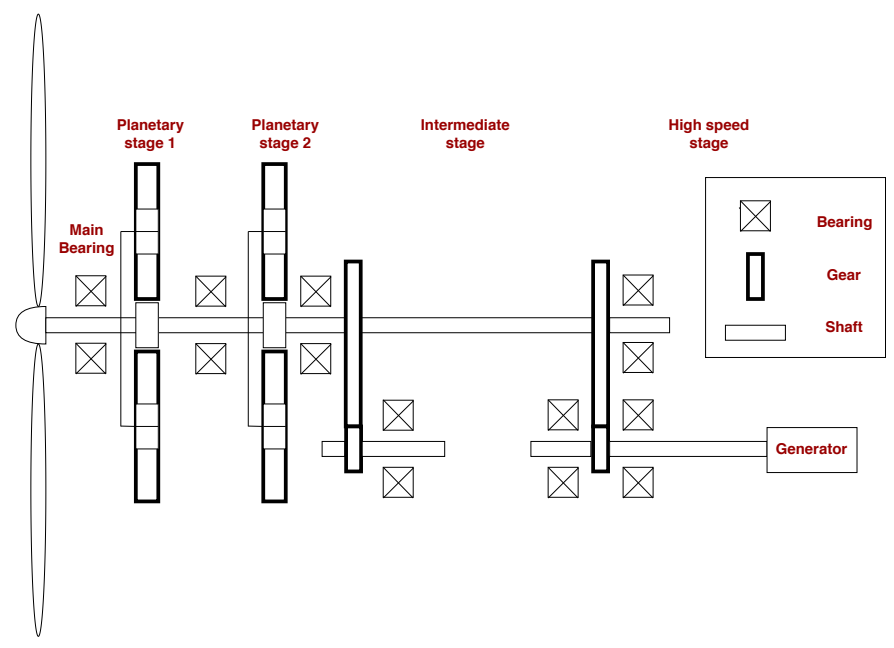

Figure 2. Common Wind Turbine Gearbox Configuration

quency and its harmonic, together with the sidebands around them are of great importance in the corresponding fault diagnosis (Wang, Han, Chu, \& Feng, 2019). Table 1 summarizes the equations of fault characteristic frequencies of different localized faults and the distribution rules of these fault characteristic frequencies in the corresponding spectrum.

\subsection{Vibration Signal Processing}

The raw vibration signal is collected using accelerometers and order tracking needs to be applied in order to remove speed fluctuations (Fyfe \& Munck, 1997). The physics of failure of gears and bearings are well documented in the literature. The various fault frequencies of components are presented in the previous section. Gear faults are reflected through amplitude and frequency modulation in the vibration signal (McFadden, 1986) whereas bearing faults excite high frequency resonances (Randall \& Antoni, 2011).

There exist various signal processing diagnostic methods (Randall \& Antoni, 2011). Diagnosis through vibration signals can be performed using time, frequency or timefrequency methods (Jardine, Lin, \& Banjevic, 2006), with the two latter having shown the most promising results since they make it easier for specific frequency components of interest to be identified. Depending on the fault location and failure mode examined, different signal processing techniques can be more effective. The paper proposes a set of parallel signal processing pipelines to reveal potential fault signatures. These are as follows:

- Fast Fourier Transform (FFT)

- Cepstrum

- Envelope Spectrum 
Table 1. Fault characteristic frequencies of different components of the planetary gearbox and the spectral lines distribution around the meshing frequency or the resonance frequency

\begin{tabular}{|c|c|c|}
\hline Component & Fault Frequency & Sideband \\
\hline PLC 1 & $f_{p l c 1}$ & - \\
\hline Sun 1 & $f_{s 1}=\left(1-\frac{Z_{r 1}}{Z_{s 1}}\right) f_{p l c 1}-f_{p l c 1}$ & $f_{s 1} Z_{s 1} \pm k f_{s 1}$ \\
\hline Planet 1 & $f_{p 1}=\frac{Z_{s 1}}{Z_{p 1}} f_{s 1}$ & $f_{s 1} Z_{s 1} \pm k f_{p 1} \pm l f_{p l c 1}$ \\
\hline Ring 1 & $f_{r 1}=\frac{Z_{s 1}}{Z_{r 1}} f_{s 1}$ & $f_{s 1} Z_{s 1} \pm k f_{r 1}$ \\
\hline \multirow[t]{3}{*}{ PLC 1 Bearing } & $f_{p l c 1, o}=\frac{P}{B}\left(1-\frac{B}{P} \cos (\theta)\right)$ & $f_{n} \pm k f_{p l c 1, o}$ \\
\hline & $f_{p l c 1, i}=\frac{n_{p l c 1}}{2}\left(1+\frac{B_{p l c 1}}{P_{p l c 1}} \cos \left(\theta_{p l c 1}\right)\right)$ & $f_{n} \pm k f_{p l c 1, i}$ \\
\hline & $f_{p l c 1, b}=\frac{n_{p l c 1}}{2}\left(1+\left(\frac{B_{p l c 1}}{P_{p l c 1}} \cos \left(\theta_{p l c 1}\right)\right)^{2}\right)$ & $f_{n} \pm k f_{p l c 1, b}$ \\
\hline \multirow[t]{3}{*}{ Planet 1 Bearing } & $f_{p 1, o}=\frac{n_{p 1}}{2}\left(1-\frac{B_{p 1}}{P_{p 1}} \cos \left(\theta_{p 1}\right)\right)$ & $f_{n} \pm k f_{p 1, o}$ \\
\hline & $f_{p 1, i}=\frac{n_{p 1}}{2}\left(1+\frac{B_{p 1}}{P_{p 1}} \cos \left(\theta_{p 1}\right)\right)$ & $f_{n} \pm k f_{p 1, i}$ \\
\hline & $f_{p 1, b}=\frac{P_{p 1}}{B_{p 1}}\left(1+\left(\frac{B_{p 1}}{P_{p 1}} \cos \left(\theta_{p 1}\right)\right)^{2}\right)$ & $f_{n} \pm k f_{p 1, b}$ \\
\hline PLC 2 & $f_{p l c 2}=f_{s 1}$ & - \\
\hline Sun 2 & $f_{s 2}=\left(1-\frac{Z_{r 2}}{Z_{s 2}}\right) f_{p l c 2}-f_{p l c 2}$ & $f_{s 2} Z_{s 2} \pm k f_{s 2}$ \\
\hline Planet 2 & $f_{p 2}=\frac{Z_{s 2}}{Z_{p 2}} f_{s 2}$ & $f_{s 2} Z_{s 2} \pm k f_{p 2} \pm l f_{p l c 2}$ \\
\hline Ring 2 & $f_{r 2}=\frac{Z_{s 2}^{p 2}}{Z_{r 2}} f_{s 2}$ & $f_{s 2} Z_{s 2} \pm k f_{r 2}$ \\
\hline \multirow[t]{3}{*}{ PLC 2 Bearing } & $f_{p l c 2, o}=\frac{P}{B}\left(1-\frac{B}{P} \cos (\theta)\right)$ & $f_{n} \pm k f_{p l c 2, o}$ \\
\hline & $f_{p l c 2, i}=\frac{n_{p l c 2}}{2}\left(1+\frac{B_{p l c 2}}{P_{p l c 2}} \cos \left(\theta_{p l c 2}\right)\right)$ & $f_{n} \pm k f_{p l c 2, i}$ \\
\hline & $f_{p l c 2, b}=\frac{n_{p l c 2}}{2}\left(1+\left(\frac{B_{p l c 2}}{P_{p l c 2}} \cos \left(\theta_{p l c 2}\right)\right)^{2}\right)$ & $f_{n} \pm k f_{p l c 2, b}$ \\
\hline \multirow[t]{3}{*}{ Planet 2 Bearing } & $f_{p 2, o}=\frac{n_{p 1}}{2}\left(1-\frac{B_{p l c 1}}{P_{p l c 1}} \cos \left(\theta_{p 2}\right)\right)$ & $f_{n} \pm k f_{p 2, o}$ \\
\hline & $f_{p 2, i}=\frac{n_{p 1}}{2}\left(1+\frac{B_{p 1}}{P_{p 1}} \cos \left(\theta_{p 2}\right)\right)$ & $f_{n} \pm k f_{p 2, i}$ \\
\hline & $f_{p 2, b}=\frac{P_{p 1}}{B_{p 1}}\left(1+\left(\frac{B_{p 1}}{P_{p 1}} \cos \left(\theta_{p 2}\right)\right)^{2}\right)$ & $f_{n} \pm k f_{p 2, b}$ \\
\hline HS Gear & $f_{h s g}=f_{s 2}$ & $f_{h s g} Z_{h s g} \pm k f_{h s g}$ \\
\hline HS Pinion & $f_{h s p}=f_{h s g} \frac{Z_{h s g}}{Z_{h s p}}$ & $f_{h s g} Z_{h s g} \pm k f_{h s p}$ \\
\hline \multirow[t]{3}{*}{ HS Bearing 1} & $f_{h s b 1, o}=\frac{n_{p 1}}{2}\left(1-\frac{B_{h s b 1}}{P_{h s b 1}} \cos \left(\theta_{h s b 1}\right)\right)$ & $f_{n} \pm k f_{h s b 1, o}$ \\
\hline & $f_{h s b 1, i}=\frac{n_{h s b 1}}{2}\left(1+\frac{B_{h s b 1}}{P_{h s b 1}} \cos \left(\theta_{h s b 1}\right)\right)$ & $f_{n} \pm k f_{h s b 1, i}$ \\
\hline & $f_{h s b 1, b}=\frac{P_{h s b 1}}{B_{h s b 1}}\left(1+\left(\frac{B_{h s b 1}}{P_{h s b 1}} \cos \left(\theta_{h s b 1}\right)\right)^{2}\right)$ & $f_{n} \pm k f_{h s b 1, b}$ \\
\hline \multirow[t]{3}{*}{ HS Bearing 2} & $f_{h s b 2, o}=\frac{n_{p 1}}{2}\left(1-\frac{B_{h s b 2}}{P_{h s b 2}} \cos \left(\theta_{h s b 2}\right)\right)$ & $f_{n} \pm k f_{h s b 2, o}$ \\
\hline & $f_{h s b 2, i}=\frac{n_{h s b 2}}{2}\left(1+\frac{B_{h s b 2}}{P_{h s b 2}} \cos \left(\theta_{h s b 2}\right)\right)$ & $f_{n} \pm k f_{h s b 2, i}$ \\
\hline & $f_{h s b 2, b}=\frac{P_{h s b 2}}{B_{h s b 2}}\left(1+\left(\frac{B_{h s b 2}}{P_{h s b 2}} \cos \left(\theta_{h s b 2}\right)\right)^{2}\right)$ & $f_{n} \pm k f_{h s b 2, b}$ \\
\hline
\end{tabular}


Table 2. Calculated features from processed signals and operational parameters used as inputs in the machine learning model.

\begin{tabular}{cc}
\hline Feature & Calculation \\
\hline RMS & $\sqrt{\frac{1}{N} \sum_{i=1}^{N} A(i)^{2}}$ \\
Kurtosis & $\frac{1}{N} \sum_{i=1}^{N}(A(i)-\bar{A})^{4}$ \\
& $\frac{1}{\left(\frac{1}{N} \sum_{i=1}^{N}(A(i)-\bar{A})^{2}\right)^{2}}$ \\
Crest Factor & $\frac{\max |A(i)|}{\sqrt{\frac{1}{N} \sum_{i=1}^{N} A(i)^{2}}}$ \\
Peak-to-Peak & $\operatorname{Max}(A(i))-M i n(A(i))$ \\
Root Sum of Squares & $\sqrt{\sum_{i=1}^{N}|A(i)|^{2}}$ \\
Power & Provided channel \\
Speed & Provided channel
\end{tabular}

\subsection{Feature Extraction}

After signals processing the next step is feature extraction from these signals. These features are health condition indicators. A summary of vibration condition indicators can be found in (Zhu, Nostrand, Spiegel, \& Morton, 2014).

The features calculated for this paper are descriptive statistics (e.g. RMS) and are shown in Table 2. These features are calculated based on the fault frequencies and the narrowband sidebands as presented in Table 1. As far as the FFT is considered, for gears the first 2 harmonics of the gear mesh frequency are considered, with a narrowband of up to 6 sidebands on either side. For bearings, the first 5 harmonics of the bearing fault frequency with a $5 \%$ window are considered. Regarding the cepstrum, a 5\% around the respective quefrencies is taken and regarding the envelope spectrum, the broadband statistical features are calculated.

The operating conditions have a strong effect on vibration signals, so variables such as the power and operating speed are also considered as features.

In Table 2, $A$ stands for the signal amplitude, $N$ for the number of samples and $i$ for the data point.

\subsection{Pattern recognition}

The aforementioned features are used as inputs in pattern recognition models. There exists a wide variety of supervised machine learning models that the interested reader can choose from (Friedman, Hastie, \& Tibshirani, 2001).

When new data samples become available, they are processed in a similar way as the historic signals used for training and the features extracted are used as inputs in the trained pattern recognition model. The output of the model is the classification of the sample that informs whether the gearbox is healthy or where a potential failure is located. The layers of the pattern recognition model are presented in the rest of the section.

The first layer of the pattern recognition model is fault detection. When not enough faulty examples are available,
Table 3. Possible gearbox fault locations and failure modes.

\begin{tabular}{ll}
\hline Fault Location & Failure Mode \\
\hline Sun 1 & Tooth Crack \\
Planet 1 & Tooth Wear \\
Ring 1 & \\
Ring 2 & \\
Planet 2 & \\
Sun 2 & \\
High Speed Gear & \\
High Speed Pinion & Outer Race Defect \\
Planet Carrier 1 Bearing & Inner Race Defect \\
Planet 1 Bearing & Rolling Element Defect \\
Planet Carrier 2 & \\
Planet Carrier 2 Bearing & \\
Planet 2 Bearing & \\
High Speed Gear Bearing & \\
High Speed Pinion Bearing & \\
\hline High Speed Shaft & Misalignment
\end{tabular}

anomaly detection models are trained based on normal operating conditions. These models can be one-class Support Vector Machines (SVMs). When a fault occurs, the outliers are outside the normal boundaries and this is flagged as an anomaly. This step is particularly useful in real world industrial applications where fault case data are often unavailable or scarce.

If a fault is present based on the anomaly detection classifier and when historic fault examples are available, models can be trained using labeled faulty data. These models are trained based on historical data, with the aim of performing gearbox incipient fault isolation and diagnosis. The model is essentially a set of two multi class classifiers. The first classifier gives the fault location and the second classifier gives the failure mode.

The potential fault locations and failure modes (and therefore the classes of the classifiers) are shown in Table 3.

\section{Case Study}

43 wind turbines with confirmed gearbox faults are considered in this case study. The wind turbines are offshore, located in 7 different wind farms, same model and are rated between 2 and $4 \mathrm{MW}$. The gearbox examined is the same type as shown in Figure 2. The confirmed gearbox fault locations presented in this case study are located on the high speed stage and are as follows:

- Generator bearing

- General damage

- High speed bearing type 1 , generator side

- Inner race defect

- Outer race defect

- Rolling element defect

- High speed bearing type 2, generator side 
- Inner race defect

- Outer race defect

- Rolling element defect

- High speed bearing, rotor side

- Inner race defect

- High speed pinion

- Tooth wear

- Tooth crack

- Intermediate speed pinion

- Tooth crack

The signals are collected from an accelerometer positioned on the top of the planetary stage in the gearbox.

In mechanical components, like gears and bearings, the degradation can be gradual until a catastrophic failure occurs. In this case study, the historic alarms of the events are known. The labeling is done using expert knowledge, by inspecting the vibration signals and looking at when fault frequencies start to occur based on each failure example. The samples that are outside the window of the degradation period are labeled as healthy. The samples that are inside the window of the degradation period are labeled as faulty for the one class classifier. For the two layers of the multi class diagnostic classifiers, the fault location and failure mode are added as classes respectively. Often when the alarm is activated, the turbine stops. That means there are often not as many faulty examples as healthy and that in general the dataset is unbalanced for most faults. Stratified cross validation is used therefore.

An example of two vibration signals, one healthy and one with a high speed pinion fault is shown in Figure 3.

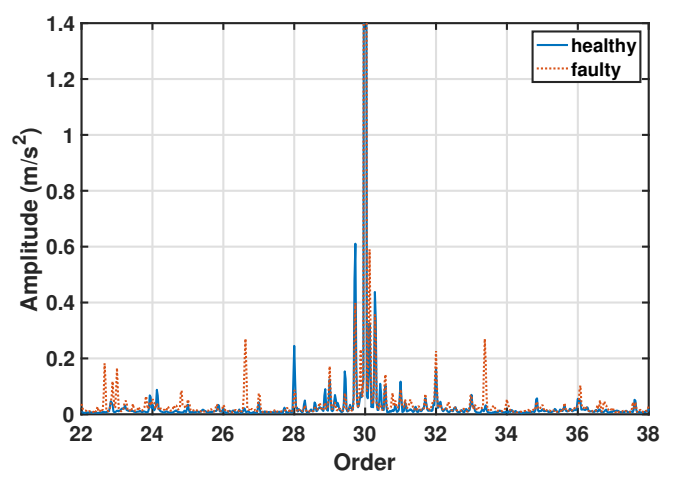

Figure 3. Order spectra of healthy and faulty signals

For the one-class classification, A one-class SVM classifier (Cortes \& Vapnik, 1995) is trained. SVMs offer superior generalisation capabilities (Widodo \& Yang, 2007). It is a semisupervised algorithm that learns a decision boundary using only normal data points and then testing the likelihood of a
Table 4. One-class classification outlier detection

\begin{tabular}{|l|l|}
\hline Precision & $87 \%$ \\
\hline Recall & $91 \%$ \\
\hline
\end{tabular}

test instance being within the boundary of the learnt model. The results of the one class classification are shown in Table 4 . The precision and recall scores indicate an adequate anomaly detection capability.

The pattern recognition model used for multi class classification is RUSboosted decision trees because they have proven to be a robust classifier that alleviates the problem of imbalanced classes (Seiffert, Khoshgoftaar, Van Hulse, \& Napolitano, 2009). In the present case study there are various types of fault examples used, however the fault classes have unequal number of samples since some failures were encountered in more wind turbines than others. RUSBoost is an algorithm that combines boosting and data sampling. It applies RUS, which is a technique that randomly removes examples from the majority class. The hyperparameters tuned are the maximum number of splits, the number of learnering cycles and the learning rate. These hyperparameters are tuned using random grid search and cross validation. The results of the stratified cross validation are shown in normalised confusion matrices in Tables 5-7.

Based on Table 5, the fraction of signals classified correctly based on their fault locations is satisfactory, and in most cases more than $80 \%$. The failure exampled that have scarce samples (like the intermediate speed pinion) are still more prone to missclassifications. On second layer of classifiers the output the failure mode and Tables 6, 7 indicate that the inner from the outer race bearing defect is more easily distinguishable than the tooth crack and wear incidents on gears. This can be justified because the symptoms for tooth cracks and wears have very similar effect on vibration signals, whereas bearing fault frequencies between inner and outer race are distinctively different.

To introduce a certain robustness cumulative counting of the classified spectra has been introduced. This illustrated for a high speed bearing and a high speed gear fault that were not included in the training set and is shown in Figures 4,5. Each time a the classification model outputs a fault, the counter adds up to this specific fault category. If another fault (or no fault) is predicted in the next sample, the counter reduces. In Figure 4, the counter starts increasing before the actual start of the alarm. After the bearing is replaced and the system is assumed to return to normal operating condition, the counter starts decreasing. In Figure 5, the classifier does not detect the fault earlier than the actual alarm which could be attribute to either the scarcity of training data for this fault location or to the fact that the fault had a sudden development. 


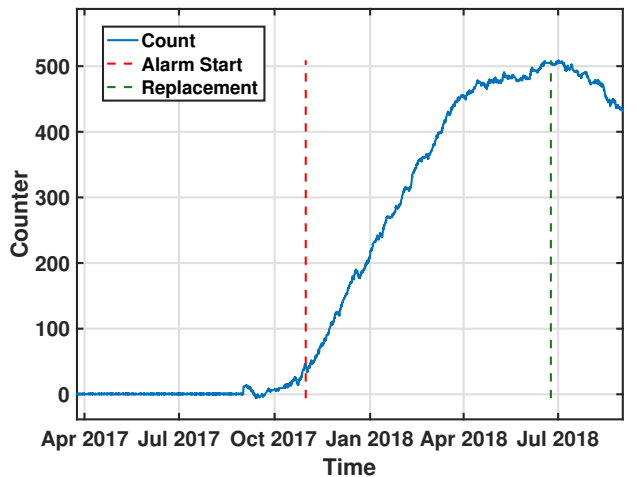

Figure 4. Counter for high speed bearing fault

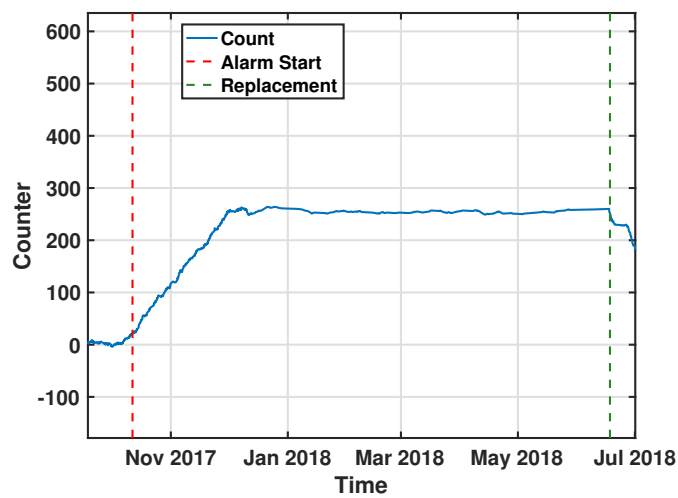

Figure 5. Counter for high speed gear fault
Table 5. Normalised confusion matrix with fault location

\begin{tabular}{|c|c|c|c|c|c|c|}
\hline $\begin{array}{c}\text { Gen } \\
\text { bearing }\end{array}$ & $\begin{array}{l}297 \\
65 \%\end{array}$ & $\begin{array}{c}70 \\
15 \%\end{array}$ & $\begin{array}{c}3 \\
1 \%\end{array}$ & $\begin{array}{c}77 \\
17 \%\end{array}$ & $\begin{array}{c}10 \\
2 \%\end{array}$ & $\begin{array}{c}0 \\
0 \%\end{array}$ \\
\hline $\begin{array}{c}\text { HS } \\
\text { bearing } \\
\text { type } 1, \\
\text { gen side }\end{array}$ & $\begin{array}{l}8 \\
<1 \%\end{array}$ & $\begin{array}{c}32259 \\
90 \%\end{array}$ & $\begin{array}{l}65 \\
<1 \%\end{array}$ & $\begin{array}{l}3171 \\
9 \%\end{array}$ & $\begin{array}{l}440 \\
<1 \%\end{array}$ & $\begin{array}{c}1 \\
1 \%\end{array}$ \\
\hline $\begin{array}{c}\text { HS } \\
\text { bearing } \\
\text { type } 2, \\
\text { gen side }\end{array}$ & 0 & $\begin{array}{l}312 \\
11 \%\end{array}$ & $\begin{array}{l}2144 \\
79 \%\end{array}$ & $\begin{array}{l}234 \\
8 \%\end{array}$ & $\begin{array}{c}54 \\
2 \%\end{array}$ & 0 \\
\hline $\begin{array}{c}\text { HS } \\
\text { bearing, } \\
\text { rotor side }\end{array}$ & $\begin{array}{l}7 \\
<1 \%\end{array}$ & $\begin{array}{c}2733 \\
6 \%\end{array}$ & $\begin{array}{l}102 \\
<1 \%\end{array}$ & $\begin{array}{c}43666 \\
93 \%\end{array}$ & $\begin{array}{l}642 \\
1 \%\end{array}$ & 0 \\
\hline$\underset{\text { pinion }}{\text { HS }}$ & 0 & $\begin{array}{l}851 \\
7 \%\end{array}$ & $\begin{array}{l}27 \\
<1 \%\end{array}$ & $\begin{array}{l}1042 \\
8 \%\end{array}$ & $\begin{array}{l}11016 \\
85 \%\end{array}$ & 0 \\
\hline \multirow[t]{2}{*}{$\begin{array}{c}\text { IMS } \\
\text { pinion }\end{array}$} & 0 & $\begin{array}{c}8 \\
12 \%\end{array}$ & $\begin{array}{c}1 \\
1 \%\end{array}$ & $\begin{array}{c}9 \\
14 \%\end{array}$ & $\begin{array}{c}8 \\
12 \%\end{array}$ & $\begin{array}{c}33 \\
60 \%\end{array}$ \\
\hline & $\begin{array}{c}\text { Gen } \\
\text { bearing }\end{array}$ & $\begin{array}{c}\text { HS } \\
\text { bearing } \\
\text { type 1, } \\
\text { gen side }\end{array}$ & $\begin{array}{c}\text { HS } \\
\text { bearing } \\
\text { type } 2 \text {, } \\
\text { gen side }\end{array}$ & $\begin{array}{c}\text { HS } \\
\text { bearing, } \\
\text { rotor side }\end{array}$ & $\underset{\text { pinion }}{\text { HS }}$ & $\underset{\text { pinion }}{\text { IMS }}$ \\
\hline
\end{tabular}

Table 6. Normalised confusion matrix with failure mode for high speed bearing

\begin{tabular}{|l|c|c|c|}
\hline Inner & 39809 & 2989 & 618 \\
Race & $92 \%$ & $7 \%$ & $1 \%$ \\
\hline Outer & 88 & 3152 & 7 \\
Race & $3 \%$ & $97 \%$ & $<1 \%$ \\
\hline Rolling & 10 & 3 & 474 \\
Element & $2 \%$ & $1 \%$ & $97 \%$ \\
\hline & Inner & $\begin{array}{l}\text { Outer } \\
\text { Race }\end{array}$ & $\begin{array}{l}\text { Rolling } \\
\text { Element }\end{array}$ \\
\hline
\end{tabular}

Table 7. Normalised confusion matrix with failure mode for high speed gear

\begin{tabular}{|l|c|c|}
\hline Tooth & 100 & 21 \\
Crack & $83 \%$ & $17 \%$ \\
\hline Tooth & 102 & 2521 \\
Wear & $4 \%$ & $96 \%$ \\
\hline & $\begin{array}{l}\text { Tooth } \\
\text { Crack }\end{array}$ & $\begin{array}{l}\text { Tooth } \\
\text { Wear }\end{array}$ \\
\hline
\end{tabular}

\section{Conclusion}

This paper presented a complete fault detection and isolation framework for wind turbine gearboxes. Vibration signals are collected from accelerometers placed on the gearbox and processed using pipelines signal processing algorithms. Health indicators are extracted and used as inputs in 3 layers of pattern recognition models; the first one indicates the health state of the gearbox, the second one the fault location (if a fault is detected in the first layer) and the third one outputs the failure mode, based on the fault location of the previous classifier layer.

The methodology is validated using a vibration signals from operating wind turbines with confirmed gearbox faults. It seems that the failure prediction results for health estimation 
and fault isolation are quite promising with a high classification rate. The failure mode classification can prove to be more challenging, especially in cases of faults that have similar symptoms. The problem of class imbalance and unequal distribution of fault examples across the dataset makes it more challenging to create and validate a robust algorithm. This signifies the need for a larger pool of failure examples to train these models.

The main limitation of this methodology is that fault cases from all locations across the gearbox are needed to train all layers of patter recognition diagnostic models. It is often the case in real operational assets that this dataset might not be available. Therefore, in this case the anomaly detection step of the framework can be applied.

More failure examples from different wind turbines should be tested and the generalization in new machines should be proven before this methodology is applied full scale.

\section{ACKNOWLEDGMENT}

The authors would like to acknowledge EPSRC funding number EP/L016680/1 for the funding of this project.

\section{REFERENCES}

Amirat, Y., Benbouzid, M. E. H., Al-Ahmar, E., Bensaker, B., \& Turri, S. (2009). A brief status on condition monitoring and fault diagnosis in wind energy conversion systems. Renewable and sustainable energy reviews, 13(9), 2629-2636.

Artigao, E., Martín-Martínez, S., Honrubia-Escribano, A., \& Gómez-Lázaro, E. (2018). Wind turbine reliability: A comprehensive review towards effective condition monitoring development. Applied energy, 228, 15691583.

Cortes, C., \& Vapnik, V. (1995). Support-vector networks. Machine learning, 20(3), 273-297.

Friedman, J., Hastie, T., \& Tibshirani, R. (2001). The elements of statistical learning (Vol. 1) (No. 10). Springer series in statistics New York, NY, USA:.
Fyfe, K., \& Munck, E. (1997). Analysis of computed order tracking. Mechanical Systems and Signal Processing, 11(2), 187-205.

Hameed, Z., Hong, Y., Cho, Y., Ahn, S., \& Song, C. (2009). Condition monitoring and fault detection of wind turbines and related algorithms: A review. Renewable and Sustainable energy reviews, 13(1), 1-39.

Jardine, A. K., Lin, D., \& Banjevic, D. (2006). A review on machinery diagnostics and prognostics implementing condition-based maintenance. Mechanical systems and signal processing, 20(7), 1483-1510.

Márquez, F. P. G., Tobias, A. M., Pérez, J. M. P., \& Papaelias, M. (2012). Condition monitoring of wind turbines: Techniques and methods. Renewable Energy, 46, 169178.

McFadden, P. (1986). Detecting fatigue cracks in gears by amplitude and phase demodulation of the meshing vibration. Journal of vibration, acoustics, stress, and reliability in design, 108(2), 165-170.

Randall, R. B., \& Antoni, J. (2011). Rolling element bearing diagnosticsa tutorial. Mechanical systems and signal processing, 25(2), 485-520.

Seiffert, C., Khoshgoftaar, T. M., Van Hulse, J., \& Napolitano, A. (2009). Rusboost: A hybrid approach to alleviating class imbalance. IEEE Transactions on Systems, Man, and Cybernetics-Part A: Systems and Humans, 40(1), 185-197.

Wang, T., Han, Q., Chu, F., \& Feng, Z. (2019). Vibration based condition monitoring and fault diagnosis of wind turbine planetary gearbox: A review. Mechanical Systems and Signal Processing, 126, 662-685.

Widodo, A., \& Yang, B.-S. (2007). Support vector machine in machine condition monitoring and fault diagnosis. $\mathrm{Me}$ chanical systems and signal processing, 21(6), 25602574.

Zhu, J., Nostrand, T., Spiegel, C., \& Morton, B. (2014). Survey of condition indicators for condition monitoring systems. In Annu. conf. progn. heal. manag. soc (Vol. 5, pp. 1-13). 